\title{
Risk Factors for Peritonitis in a Pediatric Dialysis Program in Guatemala, 2011-2014
}

\author{
Jeda Chinchilla, ${ }^{1}$ Karla Sebastián, ${ }^{1}$ Renato Meléndez, ${ }^{2}$ \\ Brooke Ramay, ${ }^{3}$ and Randall Lou-Meda ${ }^{1}$ \\ ${ }^{1}$ Fundación para el Niño Enfermo Renal, Calzada Roosevelt y 5ta. Calle, Zona 11, 01011 Ciudad de Guatemala, Guatemala \\ ${ }^{2}$ Universidad Francisco Marroquín, Calle Manuel F. Ayau (6 Calle final), Zona 10, 01010 Guatemala City, Guatemala \\ ${ }^{3}$ Universidad del Valle de Guatemala, 18 Avenida 11-95, Zona 15, 01015 Guatemala City, Guatemala \\ Correspondence should be addressed to Jeda Chinchilla; jedachinchilla88@gmail.com
}

Received 15 October 2015; Revised 29 January 2016; Accepted 16 February 2016

Academic Editor: Deepak Malhotra

Copyright (C) 2016 Jeda Chinchilla et al. This is an open access article distributed under the Creative Commons Attribution License, which permits unrestricted use, distribution, and reproduction in any medium, provided the original work is properly cited.

\begin{abstract}
Background. Peritonitis is the most common complication of peritoneal dialysis but there is limited data regarding peritonitis related risk factors in developing countries. Objective. Describe the PD program at Foundation for Children with Kidney Disease (FUNDANIER), in Guatemala, and identify peritonitis related risk factors in these patients. Methods. This retrospective open cohort study included medical records from FUNDANIER during 2011 to 2014. Baseline demographics, treatment modalities, caregivers' characteristics, and socioeconomic status were recorded. Results. Eighty-nine medical records were included with a treatment time of 1855 months. Median age of patients was 11.3 years (range 6-17). Median duration of PD therapy was 20.8 months (range 1-28). Sixty-eight peritonitis episodes were registered; forty-eight patients (54\%) remained peritonitis-free. Median time to first peritonitis episode was 5 months (range 2-16). Peritonitis rate was one episode every 27 months or 0.44 episodes per patient-year. Peritonitis rate in patients with fair housing was 2.5 times higher than in those with good housing $(\mathrm{CI}=1.0-5.2, p=0.01)$. Conclusion . Housing conditions are a relevant risk factor related to peritonitis. Strategies toward preventing peritonitis must consider housing status, establishing adequate follow-up in high-risk patients. Close monitoring of technique serves to overcome understaffing issues in this setting.
\end{abstract}

\section{Introduction}

Modality for renal replacement therapy (RRT) in patients with CKD is highly dependent on the availability of healthcare resources $[1,2]$. Peritoneal dialysis is the primary mode of treatment of pediatric RRT [2,3], the technique of choice in developing countries, and has been the primary mode of RRT for the past years in United States, Canada, Mexico, and Costa Rica nephrology centers [3,4]. Around $59-65 \%$ of patients on RRT, less than 20 years old, and up to $87 \%$, under 5 years old, use PD [3-5].

Infectious, mechanical, and metabolic complications have been described in RRT [6]. Peritonitis is the most common complication of PD in children and adults [7]. The reported annual rate of peritonitis in children using PD is 0.77-2.8 episodes per patient-year or one episode of peritonitis every $4.3-18$ patient-months [3, 7-9] having both short and long term impacts $[1,3,8]$. Peritonitis is the primary cause of catheter loss and withdrawal from dialysis treatment [10]. Peritonitis also presents a mortality risk of at least $1 \%$ and is the leading cause of death in children and adults on chronic PD $[8,11,12]$. It also presents a risk in low income countries where infection is more prevalent due to lower socioeconomic status and housing levels [7].

Development of peritonitis has been attributed to several risk factors: patients' age, time on treatment, use of automatized peritoneal dialysis (APD), exit site infections, and residual renal function [5]. Socioeconomic circumstances have shown having the greatest related risk factors on the development of peritonitis $[3,5,7]$. Outcomes of patients treated with $\mathrm{PD}$ improve by identifying risk factors and implementing specific preventive measures to overcome PD in this setting [13]. 
TABLE 1: Characteristics in common of included and not included patients.

\begin{tabular}{|c|c|c|c|}
\hline & $\begin{array}{c}\text { Included } \\
n=89\end{array}$ & $\begin{array}{l}\text { Not included } \\
\quad n=75\end{array}$ & $p$ value \\
\hline Median age (years) & 11.3 & 11.3 & \\
\hline $\begin{array}{l}\text { Patients below } 5 \text { years } \\
\text { old, } n(\%)\end{array}$ & $1(\%)$ & $2(\%)$ & 0.46 \\
\hline Sex, female & $49(55)$ & $39(29)$ & 0.69 \\
\hline Peritonitis, yes & $46(41)$ & $41(31)$ & 0.70 \\
\hline
\end{tabular}

Guatemala has recently developed the capacity to treat children with CKD through its public healthcare system. Foundation for Children with Kidney Disease (FUNDANIER) was founded in Guatemala in 2003 by parents of patients and is the only exclusive pediatric RRT program in the country which inpatients and outpatients from all regions of Guatemala attend. FUNDANIER plays a major role as a driving force facilitating changes in the Guatemalan healthcare system establishing a comprehensive pediatric nephrology program [14-16]. PD is the main treatment modality reported in FUNDANIER and it is received by $40.4 \%$ of patients $(n=193)$, followed by hemodialysis $(26.4 \%)$, transplant $(12.4 \%)$, and no renal replacement therapy $(17.6 \%)$ [17].

In countries of limited resources, specifically in Latin America, there is inadequate data surrounding peritonitis and risk factors in pediatric patients. In this study, we collected data of patients on peritoneal dialysis in order to identify risk factors associated with peritonitis.

\section{Subjects and Methods}

2.1. Study Design. We carried out a retrospective open cohort study of patients treated with peritoneal dialysis at FUNDANIER, Hospital Roosevelt, Guatemala.

2.2. Subjects. We included medical records from all patients from 0 to 18 years old, enrolled in pediatric PD program at FUNDANIER during years 2011 to 2014. Patients with incomplete demographic data or incomplete PD related information in medical records were excluded. Excluded patients were very similar in age (median 11.3 years), $41 \%$ of patients had peritonitis, and $38.7 \%$ were female. In Table 1 , we showed a comparison of included and not included patients. There is no statistically significant difference between excluded and included patients in our study.

2.3. Data Collection. Data were collected from medical records of FUNDANIER database using a standardized checklist.

2.4. Methods. Baseline data was recorded including patients' age at the beginning of the treatment, gender, chronic nutritional status (expressed as $z$-scores of height for age, classified as normal \pm 1 SD or as stunting \pm 2 SD), and the presence or absence of comorbidities.
We also sought for characteristics of patients' caregivers: spoken language, relationship with patient, literacy, and PD training results. At the beginning of the PD treatment, each patient's caregiver is required to take a five-day training course including a total of four hours per day of theoretical and practical knowledge (based on the international training course described by Baxter and in the training book for patients by Grandillo, J; et al. Manual de Capacitación del Paciente en Diálisis Peritoneal, not copyright protected, 5K9765, Baxter, Mexico) and is assessed at the end of the program. The caregiver is retrained for a second 5-day course if a failure in the technique is detected at the end of initial assessment. Retrained caregivers were classified as caregivers with "unsatisfactory training" and caregivers without a need of further training as caregivers with "satisfactory training." In addition, we recorded caregivers SES characteristics: (1) housing assessment, evaluated on the availability of electricity, water services, and toilet (good: three services, fair: two or less services); (2) place of residence (urban, rural); (3) family situation (integrated or broken); (4) employment status.

Recorded data also included time in PD treatment (months from the date the patient was enrolled to December 31st, 2014, or the date patient left PD program) and treatment modality (continuous ambulatory PD (CAPD) or automatized PD (APD)).

Peritonitis episodes were recorded. All episodes were diagnosed by a pediatric nephrologist and were documented in the medical chart using a standardized log sheet identifying characteristics of each peritonitis episode. Peritonitis outcome indexes were calculated: (1) peritonitis rate (expressed as one peritonitis episode for every number of patientmonths and as the number of episodes/patient-year); (2) peritonitis-free period (time to first peritonitis episode); (3) peritonitis-free patients (patients who did not present with peritonitis during study period).

2.5. Statistical Analysis. Parametric data are presented as mean and standard deviation (SD). Nonparametric data are presented as median values and Interquartile Range (IQR). Peritonitis rate ratios and peritonitis-free period were calculated as mentioned before. Peritonitis rate was calculated for each risk factor (exposed and unexposed). Incidence Rate Ratio (IRR) was used to assess possible association between each risk factor and peritonitis episodes. Statistical analysis was performed using STATA 13.1 software. We indicated significance with a value of $p$ less than 0.05 .

\section{Results}

Out of the 182 medical records reviewed, 164 were enrolled between 2011 and 2014 and 89 (48.9\%) met inclusion criteria. Distribution of gender, age, and presence of peritonitis episodes in excluded patients were similar with those of included patients. In total, records represented 1855 patientmonths of PD therapy.

3.1. Patient Characteristics. Baseline data is shown in Table 2. The most important comorbidity additional to ESRD out of 
TABLE 2: Demographic data and potential risk factors for peritonitis in pediatric patients on peritoneal dialysis, $N=89$.

\begin{tabular}{|c|c|c|c|c|c|c|}
\hline & $n(\%)$ & Patients with peritonitis, $n(\%)$ & Peritonitis rate ${ }^{* *}$ & $\mathrm{IRR}^{* * *}$ & $p^{+}$ & $\mathrm{CI}$ \\
\hline \multicolumn{7}{|l|}{ Gender } \\
\hline Male & $40(44.9)$ & $18(45.0)$ & 0.50 & \multirow{2}{*}{0.91} & \multirow{2}{*}{0.35} & \multirow{2}{*}{$0.5-1.5$} \\
\hline Female & $49(55.1)$ & $23(46.9)$ & 0.42 & & & \\
\hline \multicolumn{7}{|l|}{ Nutritional status } \\
\hline Normal & $28(31.5)$ & $13(46.4)$ & 0.60 & \multirow{2}{*}{0.70} & \multirow{2}{*}{0.08} & \multirow{2}{*}{$0.4-1.2$} \\
\hline Stunting & $61(68.5)$ & $28(45.9)$ & 0.40 & & & \\
\hline \multicolumn{7}{|l|}{ Comorbidity } \\
\hline No & $77(86.5)$ & $36(46.7)$ & 0.46 & \multirow{2}{*}{0.71} & \multirow{2}{*}{0.17} & \multirow{2}{*}{$0.3-1.4$} \\
\hline Yes & $12(13.5)$ & $5(41.7)$ & 0.33 & & & \\
\hline \multicolumn{7}{|l|}{ Place of residence ${ }^{*}$} \\
\hline Urban & $47(52.8)$ & $20(42.5)$ & 0.43 & \multirow{2}{*}{1.02} & \multirow{2}{*}{0.45} & \multirow{2}{*}{$0.6-1.7$} \\
\hline Rural & $42(47.2)$ & $21(50.0)$ & 0.44 & & & \\
\hline \multicolumn{7}{|c|}{ Caregiver relationship ${ }^{* * * *}$} \\
\hline Parents & $68(81.0)$ & $32(47.1)$ & 0.46 & \multirow{2}{*}{0.88} & \multirow{2}{*}{0.36} & \multirow{2}{*}{$0.4-1.7$} \\
\hline Others & $16(19.0)$ & $8(50.0)$ & 0.41 & & & \\
\hline \multicolumn{7}{|l|}{ Caregiver literacy ${ }^{* * * *}$} \\
\hline Literate & $70(88.6)$ & $33(47.1)$ & 0.45 & \multirow{2}{*}{1.26} & \multirow{2}{*}{0.22} & \multirow{2}{*}{$0.6-2.4$} \\
\hline Illiterate & $9(11.4)$ & $7(77.8)$ & 0.60 & & & \\
\hline \multicolumn{7}{|l|}{ Training results ${ }^{* * * *}$} \\
\hline Satisfactory & $61(96.8)$ & $21(34.4)$ & 0.31 & \multirow{2}{*}{1.47} & \multirow{2}{*}{0.25} & \multirow{2}{*}{$0.3-4.7$} \\
\hline Unsatisfactory & $2(3.2)$ & $2(100.0)$ & 0.50 & & & \\
\hline \multicolumn{7}{|l|}{ Housing ${ }^{* * * *}$} \\
\hline Good (3 services) & $73(94.8)$ & $35(47.9)$ & 0.43 & \multirow{2}{*}{2.50} & \multirow{2}{*}{0.01} & \multirow{2}{*}{$1.0-5.2$} \\
\hline Fair ( $\leq 2$ services) & $4(5.2)$ & $3(75.0)$ & 1.06 & & & \\
\hline Family situation ${ }^{* * * *}$ & & & & & & \\
\hline Integrated & $54(66.7)$ & $27(50.0)$ & 0.46 & 0.91 & 0.37 & $0.5-1.6$ \\
\hline Broken & $27(33.3)$ & $12(44.4)$ & 0.42 & & & \\
\hline
\end{tabular}

${ }^{+} p \leq 0.05$ significant

* Place of residence: urban, department of Guatemala; rural, outside department of Guatemala.

${ }^{* *}$ Peritonitis rate: number of peritonitis episodes/treatment-years.

*** IRR: Incidence Rate Ratio, peritonitis rate exposed group/peritonitis rate unexposed group.

${ }^{* * * *}$ Underreported data: patients with incomplete information at medical records.

TABLE 3: Comorbidities of pediatric patients on peritoneal dialysis, $N=12$.

\begin{tabular}{llc}
\hline Comorbidity & $n$ & $\%$ \\
\hline Congenital heart disease & 3 & 25.0 \\
Seizures & 2 & 16.7 \\
Uropathy & 2 & 16.7 \\
Polycystic kidney disease & 2 & 16.7 \\
Asthma & 1 & 8.3 \\
Diabetes mellitus type I & 1 & 8.3 \\
Gastroesophageal reflux disease & 1 & 8.3 \\
\hline
\end{tabular}

the $12(13.5 \%)$ patients presenting with comorbidities was congenital heart disease (25\%). Table 3 shows comorbidities that patients presented with.

3.2. Caregivers. Spoken language was not included in Table 1 since most of caregivers ( $n$ 88, 98.9\%) spoke Spanish. Data was underreported for the rest of characteristics: relationship with patient $(n 75,94.4 \%)$, literacy $(n 79,88.8 \%)$, PD training results ( $n$ 63,70.8\%), housing ( $n 77,86.5 \%)$, family situation $(n 81,91.0 \%)$, and employment status ( $n 70,78.6 \%)$. Since most caregivers had "satisfactory training" (96.8\%), this characteristic was neither included in Table 1.

3.3. Socioeconomic Status. Seventy-three patients had "good housing" (94.8\%) and four had "fair housing" (5.2\%).

\subsection{Peritoneal Dialysis Treatment and Peritonitis Prevalence}

3.4.1. Peritoneal Dialysis Treatment. Median age at the beginning of PD treatment was 11.3 years old (range 6-17) and median time on PD was 20.8 months (range 1-28). Fiftyeight patients were in PD for more than 12 months (65.2\%). CAPD was the most common modality of RRT used by participants $(n 84,94.4 \%)$. Catheters used in all the patients were Pediatric Curl Cath for peritoneal dialysis with one 
TABle 4: Peritoneal fluid cultures corresponding to peritoneal dialysis related episodes of peritonitis in pediatric patients, $N=48$.

\begin{tabular}{lcc}
\hline Germ isolated & Number of cultures & $\%$ \\
\hline Staphylococcusaureus & 20 & 41.7 \\
Sterile & 14 & 29.2 \\
Pseudomonasaeruginosa & 6 & 12.5 \\
Acinetobacterbaumannii & 2 & 4.2 \\
Serratiamarcescens & 2 & 4.2 \\
Candidaparapsilosis & 1 & 2.1 \\
Pseudomonasputida & 1 & 2.1 \\
Burkholderiacepacia & 1 & 2.1 \\
Staphylococcusepidermidis & 1 & 2.1 \\
\hline
\end{tabular}

felt cuff (Kendall Quinton, Tyco Healthcare). All patients were on PD treatment as a result of ESRD. Included records represented 1855 patient-months of PD therapy.

3.4.2. Peritonitis Prevalence and Outcome Indexes. During the study period, a total of 41 patients were shown to have at least one episode of peritonitis (46\%). Within the study period, 68 episodes of peritonitis were registered. Forty-eight patients had no reports of peritonitis (54\%). A total of 48 peritoneal fluid samples were sent to microbiology laboratory for cultures (Table 4 ). In the 20 remaining peritonitis episodes, samples were not sent to culture because laboratory services were not available. The median number of peritonitis episodes per patient was 1.66 episodes (range 1-4). Median time from the beginning of treatment to the first episode of peritonitis was 5 months (range 2-16). Peritonitis rate in the study period was one episode every 27 months or 0.44 episodes per patient-year.

\subsection{Risk Factor Analysis}

3.5.1. Peritonitis Prevalence. All potential risk factors for peritonitis prevalence were analyzed as shown in Table 1. Out of 89 patients, 41 had had at least one peritonitis episode (46\%). Distribution of peritonitis prevalence was homogeneous within the risk factor categories of gender, age, nutritional status, comorbidity and place of residence, height for age, and family situation. Prevalence of peritonitis was higher for patients who had a nonparent caregiver, an illiterate caregiver, unsatisfactory PD training, APD modality of PD, fair housing, and unemployed family status. However, these differences were not statistically significant.

3.5.2. Peritonitis Rate. There was a positive association between peritonitis rate (episodes per year) and patients greater than 10 years old, illiterate caregiver, unsatisfactory training, rural place of residence, CAPD modality, and unemployed family status. These associations, however, were not statistically significant. When good housing was compared to fair housing, the peritonitis rate was significantly lower in good housing. Peritonitis rate for fair housing was 2.50 times higher compared to peritonitis rate of good housing ( $p 0.01)$.

\section{Discussion}

This retrospective cohort study shows there was a low prevalence of peritonitis in Guatemalan pediatric population seen at FUNDANIER from 2011 to 2014. FUNDANIER is a relatively new pediatric nephrology program serving most of the Guatemalan pediatric population with ESRD. Although FUNDANIER is a relatively new program, the cohort being treated here had a longer PD treatment time (1855 patientmonths) compared to other programs with treatment periods from 578 and 544 patient-months [3, 7]. Additionally, the peritonitis rate of our program was comparable to reports from treatment centers in the developed world that have a longer history of providing PD [5]. Also, the population that remained peritonitis-free at FUNDANIER was similar to what has been reported in Vienna where $43 \%$ patients were free of peritonitis [5] and in South Africa with $28.5 \%$ patients peritonitis-free [7].

We perceive that undiagnosed cases are the result of patients arriving to clinic in the late stages of ESRD. The most common cause of ESRD in children is Congenital Anomalies of the Kidney and the Urinary Tract (CAKUT) [1]. However at FUNDANIER, the primary cause of CKD is undetermined and occurs in $43 \%$ of cases. CAKUT is the second most common cause of CKD stage 2 or greater occurring in $28 \%$ of patients. Twelve percent of cases are secondary to glomerulopathies [17]. In the cohort, one 3year-old patient was included and the underlying cause of ESRD was CAKUT.

The population of patients seen at our clinic is skewed toward older aged patients. This is most likely due to issues in access to health services, resulting in patients seeking medical attention in the late stage of ESRD, when the underlying cause cannot be identified. In this setting, the underlying cause of ESRD cannot be identified in every single patient.

At FUNDANIER 28\% of patients were reported as having CDK stage 2 or greater; of these, $35 \%$ were $10-14$ years of age and 29\% were 5-9 years of age; the number of patients with ESRD is noticeably higher in the group above 10 years of age [17]. Age of patients initiating PD treatment at FUNDANIER was similar to previous studies, where patients initiated PD at 10.1 years [7] and 11.7 years [3]. The majority of patients in our cohort were older than 10 years, correlating with the number of patients over 10 years with ESRD in Guatemala [17]. Although patient's older age was positively associated with peritonitis in our study, it was not statistically significant. These data contrast other studies where younger age has been reported as a risk factor for peritonitis [5, 18-20]. In these studies, patients less than 2 years old had more peritonitis episodes and a smaller proportion remained peritonitis-free [5]. Our data may differ from other studies because in our study older age was positively associated with peritonitis because in our study the proportion of patients less than 5 years old is underrepresented. Nevertheless, it is important to take into consideration age when initiating a PD program.

The time on PD treatment of our patients was 20.8 months, similar to a previous study in Israel, reporting a median of 19 months on PD treatment of which $55 \%$ of patients had more than 12 months of treatment experience 
[3]. In our population, the period to first peritonitis episode was longer than previously reported [3,7]. Early onset of peritonitis, in the first 6 months of treatment, has been reported as a risk factor, alerting physicians to enhance patient and caregiver training at the beginning of training [3]. Improvement of PD technique in the early months of treatment is essential to ensure patient permanence in the program, providing long term, high quality treatment. We suspect that our patients have a longer time to first peritonitis episode because patients have frequent checkups at the start of PD treatment through a multidisciplinary team that includes a social worker, psychologist, nutritionist, PD nurse, and physicians. Before patients leave the clinic, we ensure that caregivers understand how to carry out PD and are retrained if necessary reducing complications related to $\mathrm{PD}$ technique and further maximizing time to first peritonitis episode.

Patients in our study did not have frequent home visits supervising their dialysis technique, due to the fact that FUNDANIER has one social worker and one PD nurse for all patients in the program, having a low personnel-topatient rate. We acknowledge that, as a part of a successful training program, it should include home visits, which are valuable for improving clinical care in pediatric patients utilizing home PD [21]. However, we based our surveillance on the observed technique monitored at every clinic visit and/or hospitalization that patient had for any given reason. This study has shown that regular supervision of caregiver techniques is decisive for a positive PD outcome.

CAPD is the most commonly used PD modality at FUNDANIER and has a lower cost compared to APD [11]. CAPD modality at FUNDANIER had a higher reported peritonitis rate than APD, which is similar to what has been shown in other studies [22]. This may be due to the number of connection and disconnection events (fewer in APD compared to CAPD) [3, 22]. In our study, patients on APD lived in institutional households, and the caregiver was not a relative; all of these patients had at least one peritonitis episode, suggesting that there may be a relationship between peritonitis and emotional, social, and psychological factors in this setting. This may affect patient situation as is shown in one study where lack of motivation was reported as a modifiable predictor factor of peritonitis [23].

FUNDANIER is located in urban area of Guatemala and patients living in rural area have to travel long distances causing delays in receiving specialized medical care. Nevertheless, place of residence, used to measure distance to center, did not influence peritonitis rate, nor did it in previous studies $[7,24]$. Patients with unemployed family had a higher peritonitis rate, similar to other studies showing a positive relationship between socioeconomic status and peritonitis rate [7]. This shows that family employment status in Guatemala may predict peritonitis rate more than place of residence in this setting.

Our data suggest that peritonitis depends on socialenvironment factors, especially housing, compared to personal patient conditions like gender, age, nutrition, and presence of comorbidities. Peritonitis rate in our study was 1.06 episodes/patient-year in patients with fair housing, compared with 0.43 episodes/patient-year in patients with good housing. Raaijmakers et al. reported similar results, where patients with fair housing had a peritonitis rate of 3.86 episodes/patient-year and patients with good housing 2.21 episodes/patient-year ( $p$ 0.03) [7]. Shorter times to first peritonitis episode and higher peritonitis rates in patients with poor socioeconomic status and inadequate housing were also reported [7]. Fair housing provides inappropriate conditions for PD treatment. In order to prevent peritonitis episodes, it is advisable to have a home with at least basic services such as electricity, water services, and adequate sanitation. Unfortunately in many Guatemalan households there are few or none of these resources, all of which negatively affect health outcomes. Home visits ensuring availability of resources are part of the training program at FUNDANIER, where dialysis technique in the patients' environment is supervised; however, frequency of home visits by FUNDANIER social worker is not sufficient. In every home visit, the social worker should check housing conditions and adapt to PD training according to patients' environmental conditions. Frequent interactions between healthcare staff and patients on PD improve patients attitude and greater involvement in their treatment [25]. It is important that multidisciplinary teams work toward an integral PD program ensuring an optimal quality of life for the patient [26]. According to our results, home visits need to be carried out more frequently, specifically in cases in which patients have poor-housing environments, in order to ensure adequate administration of PD within the patient environment. However, when staff is limited within an institution, their optimal efforts are key in order to achieve ideal clinical results. Our study shows that regular monitoring of patients can help us overcome the limitations faced by issues in understaffing.

4.1. Limitations. The major limitation of this study was underreporting of data in the medical records, which may have reduced study power, a characteristic inherent to retrospective cohort studies. Most data presented regarding risk factors were positively associated with peritonitis but statistically insignificant. Nevertheless, these data are helpful in understanding information regarding our PD program characteristics and programs working in similar settings.

\section{Conclusions}

Peritonitis is a complication of PD, the main RRT in developing countries. Although statistically insignificant, peritonitis rate was positively associated with age, caregiver literacy, caregiver training, and economic status. Higher peritonitis rate was statistically significant for those with fair housing status, emphasizing the importance of an integral training program that considers patients' and family member's socioenvironmental conditions. Understaffing limitations were overcome in our program through close monitoring of technique during every patient visit to the center. Future programs in this and similar settings may consider taking a multidisciplinary approach toward developing home visits and regular surveillance activities as part of their PD program. 


\section{Conflict of Interests}

The authors declare that there is no conflict of interests regarding the publication of this paper.

\section{Acknowledgment}

The authors wish to thank Dr. John Christenson for his comments on the paper.

\section{References}

[1] B. A. Warady and V. Chadha, "Chronic kidney disease in children: the global perspective," Pediatric Nephrology, vol. 22, no. 12, pp. 1999-2009, 2007.

[2] J. Harambat, K. J. Van Stralen, J. J. Kim, and E. J. Tizard, "Epidemiology of chronic kidney disease in children," Pediatric Nephrology, vol. 27, no. 3, pp. 363-373, 2012.

[3] R. Cleper, M. Davidovits, Y. Kovalski, D. Samsonov, J. Amir, and I. Krause, "Peritonitis in a pediatric dialysis unit: local profile and implications," Israel Medical Association Journal, vol. 12, no. 6, pp. 348-352, 2010.

[4] M. B. Leonard, L. A. Donaldson, M. Ho, and D. F. Geary, "A prospective cohort study of incident maintenance dialysis in children: an NAPRTC study," Kidney International, vol. 63, no. 2, pp. 744-755, 2003.

[5] M. Boehm, A. Vácsei, C. Aufricht, T. Mueller, D. Csaicsich, and K. Arbeiter, "Risk factors for peritonitis in pediatric peritoneal dialysis: a single-center study," Pediatric Nephrology, vol. 20, no. 10, pp. 1478-1483, 2005.

[6] J. E. Kim, S. J. Park, J. Y. Oh et al., "Noninfectious complications of peritoneal dialysis in Korean children: a 26-year single-center study," Yonsei Medical Journal, vol. 56, no. 5, pp. 1359-1364, 2015.

[7] R. Raaijmakers, P. Gajjar, C. Schröder, and P. Nourse, "Peritonitis in children on peritoneal dialysis in Cape Town, South Africa: epidemiology and risks," Pediatric Nephrology, vol. 25, no. 10, pp. 2149-2157, 2010.

[8] B. A. Warady, "Peritonitis," in Pediatric Dialysis, B. A. Warady, F. S. Schaefer, R. N. Fine, and S. R. Alexander, Eds., pp. 393-414, Kluwer Academic, Boston, Mass, USA, 2004.

[9] G. Klaus, "Prevention and treatment of peritoneal dialysisassociated peritonitis in pediatric patients," Peritoneal Dialysis International, vol. 25, supplement 3, pp. S117-S119, 2005.

[10] G. Woodrow, J. H. Turney, and A. M. Brownjohn, "Technique failure in peritoneal dialysis and its impact on patient survival," Peritoneal Dialysis International, vol. 17, no. 4, pp. 360-364, 1997.

[11] E. Verrina, A. Edefonti, B. Gianoglio et al., "A multicenter experience on patient and technique survival in children on chronic dialysis," Pediatric Nephrology, vol. 19, no. 1, pp. 82-90, 2004.

[12] T. Sakac1, E. Ahbap, Y. Koc et al., "Clinical outcomes and mortality in elderly peritoneal dialysis patients," Clinics, vol. 70, no. 5, pp. 363-368, 2015.

[13] B. Piraino, G. R. Bailie, and J. Bernardini, "Peritoneal dialysisrelated infection recommendations: 2005 update," Peritoneal Dialysis International, vol. 25, no. 2, pp. 107-131, 2005.

[14] R. Lou-Meda, "Prevention of CKD in Guatemala," Clinical Nephrology, vol. 74, supplement 1, pp. S126-S128, 2010.

[15] R. Lou-Meda, "ESRD in Guatemala and a model for preventive strategies: outlook of the Guatemalan Foundation for children with kidney diseases," Renal Failure, vol. 28, no. 8, pp. 689-691, 2006.

[16] R. Lou-Meda, "Comprehensive approach to pediatric kidney diseases in Guatemala," Clinical Nephrology, vol. 83, no. 7, supplement 1, pp. 82-84, 2015.

[17] A. Ceron, M. P. Fort, C. M. Morine, and R. Lou-Meda, "Chronic kidney disease among children in Guatemala," Revista Panamericana de Salud Pública, vol. 36, no. 6, pp. 376-382, 2014.

[18] R. Zent, J. E. Myers, D. Donald, and B. L. Rayner, “Continuous ambulatory peritoneal dialysis: an option in the developing world?" Peritoneal Dialysis International, vol. 14, no. 1, pp. 4851, 1994.

[19] F. Schaefer, G. Klaus, D. E. Müller-Wiefel, and O. Mehls, "Current practice of peritoneal dialysis in children: results of a longitudinal survey. Mid European Pediatric Peritoneal Dialysis Study Group (MEPPS)," Peritoneal Dialysis International, vol. 19, supplement 2, pp. S445-S449, 1999.

[20] X. Fan, R. Huang, J. Wang et al., "Risk factors for the first episode of peritonitis in Southern Chinese continuous ambulatory peritoneal dialysis patients," PLoS ONE, vol. 9, no. 9, Article ID e107485, 2014.

[21] E. N. Ellis, C. Blaszak, S. Wright, and A. Van Lierop, "Effectiveness of home visits to pediatric peritoneal dialysis patients," Peritoneal Dialysis International, vol. 32, no. 4, pp. 419-423, 2012.

[22] K. S. Rabindranath, J. Adams, T. Z. Ali, C. Daly, L. Vale, and A. M. MacLeod, "Automated vs continuous ambulatory peritoneal dialysis: a systematic review of randomized controlled trials," Nephrology Dialysis Transplantation, vol. 22, no. 10, pp. 29912998, 2007.

[23] K. Paudel, G. Namagondlu, N. Samad, K. Mckitty, and S. L. Fan, "Lack of motivation: a new modifiable risk factor for peritonitis in patients undergoing peritoneal dialysis?" Journal of Renal Care, vol. 41, no. 1, pp. 33-42, 2015.

[24] M. M. Sood, P. Komenda, A. R. Sood et al., "Adverse outcomes among Aboriginal patients receiving peritoneal dialysis," Canadian Medical Association Journal, vol. 182, no. 13, pp. 1433-1439, 2010.

[25] R. Ruiz de Gauna, I. Minguela Pesquera, J. Ocharán Corcuera, I. Gimeno Martín, and A. Chena Alejandro, "The social environment of patients on peritoneal dialysis," Nefrologia, vol. 28, supplement 6, pp. 133-136, 2008.

[26] G. Elios Russo, A. Martinez, S. Mazzaferro et al., "Optimal use of peritoneal dialysis with multi-disciplinary management," Giornale Italiano di Nefrologia, vol. 30, no. 4, 2013. 


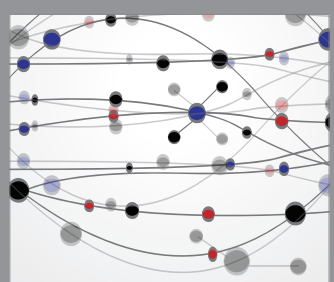

The Scientific World Journal
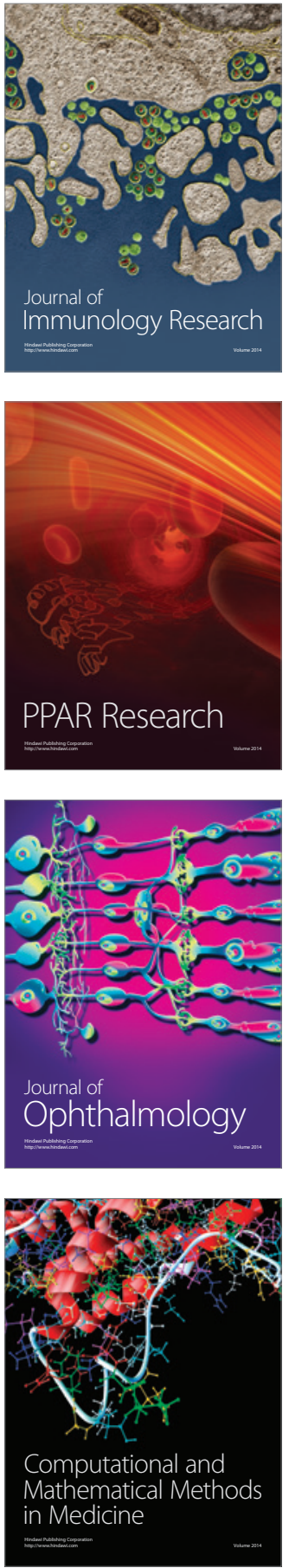

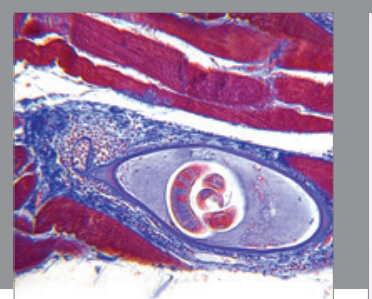

Gastroenterology Research and Practice

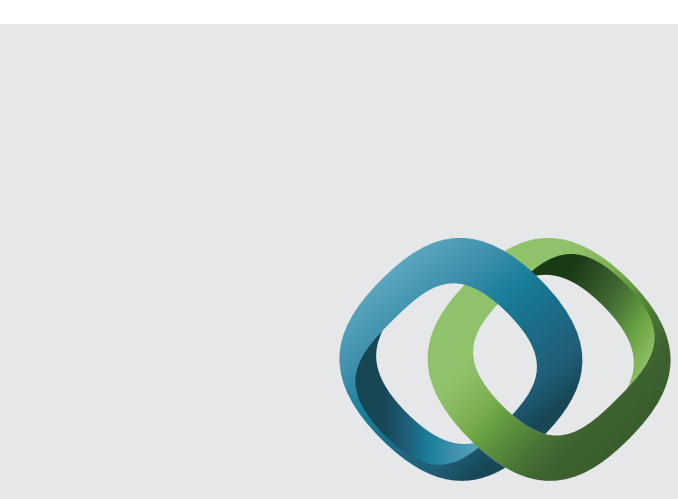

\section{Hindawi}

Submit your manuscripts at

http://www.hindawi.com
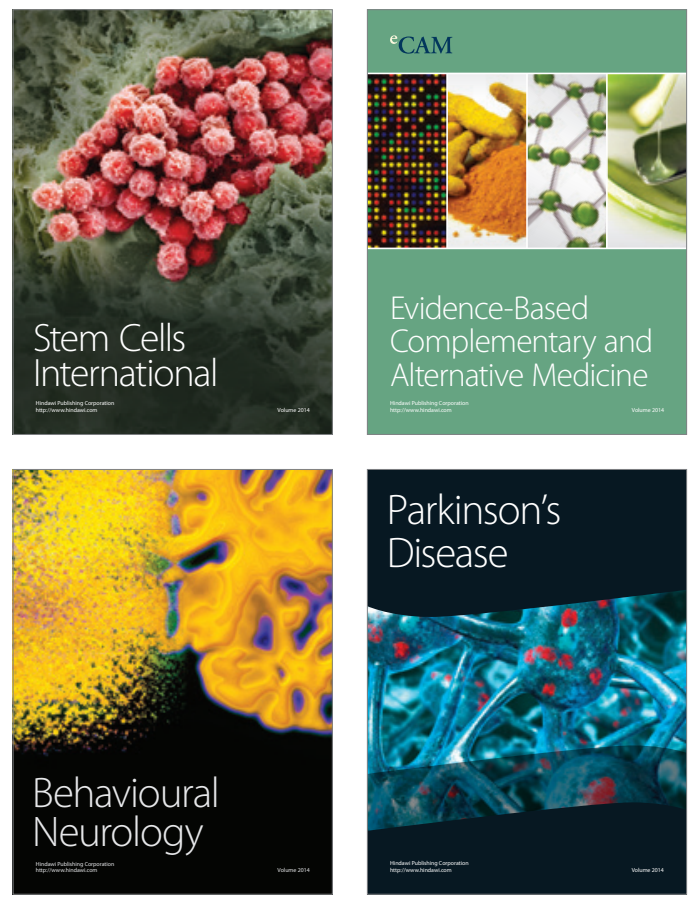
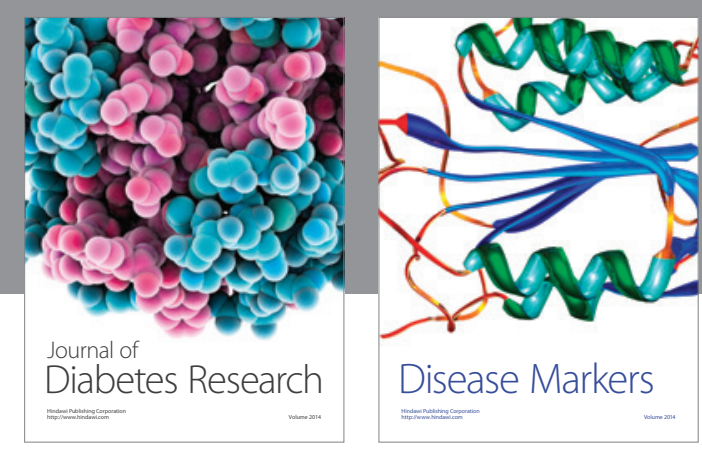

Disease Markers
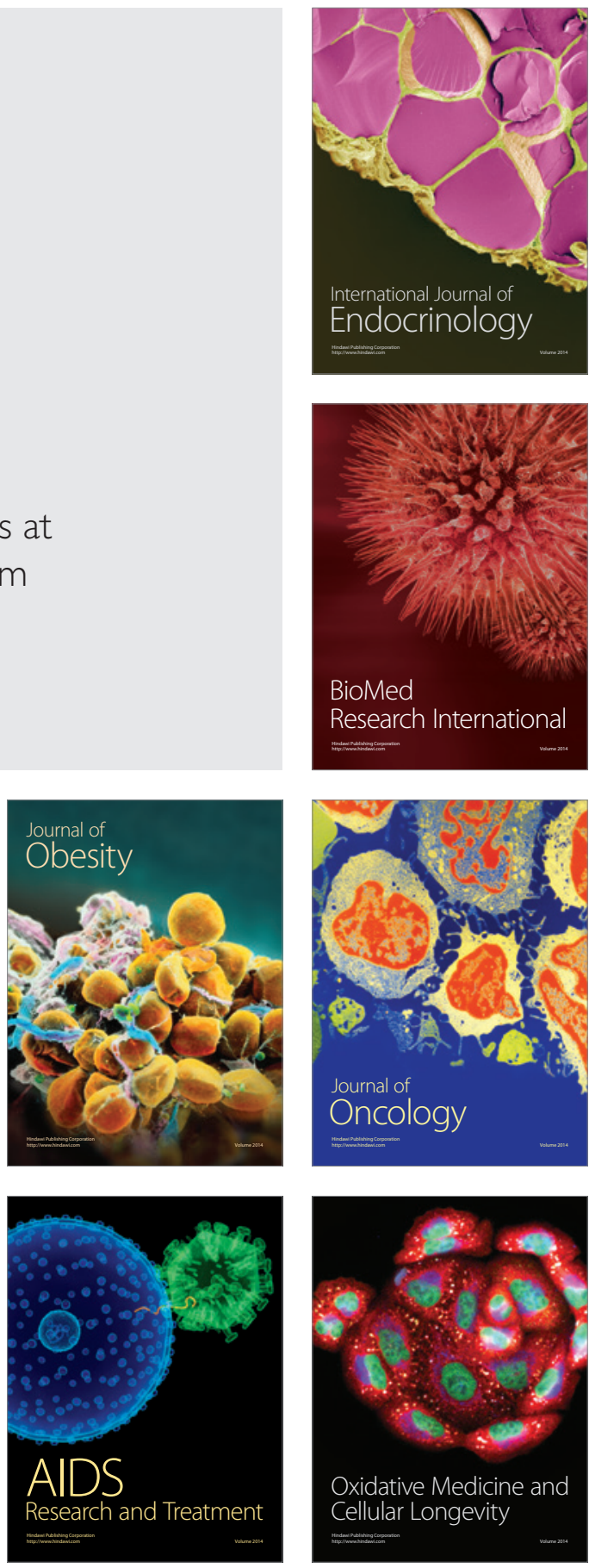